Article

\title{
The intertwined life histories and transmission ecologies of plant, arthropod, and vertebrate viruses
}

\author{
Jan Slingenbergh \\ Formerly Food and Agriculture Organization of the United Nations, 00153 Rome, Italy; \\ slingenberghj@gmail.com
}

\section{Abstract}

It remains poorly understood how the life history strategies and transmission ecologies of viruses of plants, arthropods, and vertebrates are interrelated. The present analysis hinges on the virus transmission success. Virus transmission reflects where in the host-body viruses are retained or replicating. Plants, arthropods, and vertebrates share a protective outer-layer, a circulatory system, and reproductive organs. The latter enables vertical virus transmission and associates with virus-host mutualism. Two broadly opposing virus life history strategies are considered. Acute viruses tend to be replicative and are swiftly transmitted to the next host. Instead, persistent viruses keep virus replicating costs and host damage to a minimum. The intertwined life histories and transmission ecologies are accordingly pieced together, based on the virus mono- or instead dual-host tropism, the location of virus retention or replication on or in the host-body, the presence of cyclical or mechanical transmission by arthropods, and of horizontal and vertical host-to-host transmission modes. It is shown that in the arthropod and in the vertebrate animal host, virus circulation in the hemocoel or blood circulation goes hand-in-hand with vertical transmission. Instead, plant phloem viruses do not transmit via seed. The latter is the rule for the plant-only viruses. The risk management implications are discussed in brief.

Keywords: virus life history, transmission ecology, evolution, arthropod host, virus-host mutualism 


\section{Introduction}

Making it to the next host goes to the heart of virus fitness. The virus transmission mechanisms and modes reflect where in the host-body the virus is retained or replicating [1]. There are common features to the body-plan of plants, arthropods, and vertebrates. The three host types share a protective outer-layer, an interface with the environment external to the host-body. Each avails of a circulatory system, providing virus access to all body parts. In addition, each feature reproductive organs, enabling vertical virus transmission. The latter has the effect of bringing virus and host together and associates with benign virus-host intimacy, enhanced from one host generation to the next.

There are two broadly opposing virus life history strategies. Acute viruses tend to be replicative and are swiftly transmitted to the next host. Instead, persistent viruses tend to be shed in minor quantities, if at all, keeping virus replication costs and host damage to a minimum. [2,3,4].

The feeding habits of phytophagous and hematophagous arthropods have brought a myriad of dualhost virus transmission cycles. Phylogenetic evidence supports the notion that the arthropodindependent viruses today circulating in vertebrates were ultimately derived from arthropoddependent ancestors, with subsequent adaptation to vertebrate-only transmission [5]. Arthropod viruses fall basal to vertebrate specific arenaviruses, filoviruses, hantaviruses, influenza viruses, lyssaviruses and paramyxoviruses [5]. Likewise, arthropod viruses fall basal to plant-insect-plant transmitting viruses, in particular viruses belonging to the Bunyaviridae and the Rhabdoviridae families [5].

In the present study, an attempt is made to clarify in further detail how the life histories and transmission ecologies of plants, arthropods, and vertebrate animals are intertwined. It is first recalled how insect transmitted plant viruses may be categorized on the basis of the location of virus retention or replication on or in the insect-body $[6,7,8]$. The extent of virus infiltration is matched against the duration of virus transmission. Also considered is the corresponding presence or absence of vertical transmission, in the insect and in the plant host.

Likewise, the transmission mechanisms of the world main livestock viruses are considered in further detail, 36 in total [1].

Next, the insect specificity of the insect transmitted plant viruses is explored. For this, viruses are grouped by hemipteroid family [7]. Insect specificity is considered to be reflected by the pronounced virus deep-rootedness and persistence in the insect body, shift to a single virus transmission mechanism per family, virus replication in the hemocoel, and the associated shift to vertical transmission from insect-to-insect.

The evolutionary histories and transmission ecologies of arthropod, plant, and vertebrate viruses are accordingly pieced together. Arthropods and vertebrates are distinctly more related to each other than to plants. In arthropods and vertebrates, virus replication in the hemocoel or blood circulation associates with vertical transmission. Instead, plant phloem restricted viruses are not vertically transmitted from plant-to-plant. These viruses transmit vertically in the insect host. Plant-only viruses transmit via seed. The implications for plant, animal, and public health protection are discussed in brief. 


\section{Materials and methods}

The analysis entails an iterative process. Based on the available literature it is first recalled how insect transmitted plant viruses may conveniently be categorized on the basis of four distinct virus transmission mechanisms [8]. These mechanisms reflect the extent of virus infiltration in the insectbody and the duration of virus transmission. Viruses may attach to the cuticular surfaces of the stylets, of the foregut, be retained in the salivary glands, or replicate systemically in the hemocoel. The four transmission mechanisms are described as non-persistent, semi-persistent, persistent and circulative, and persistent plus propagative. Also fitted is the virus retention time in the insect, varying from minutes to hours to days or weeks to the lifespan of the insect. In addition, the corresponding modes of transmission, both plant-insect-plant and vertical transmission in insects, are fitted. Moreover, the matching virus replication site in the plant host is shown. Virus replication may not be restricted to the phloem, for some viruses restricted to phloem for others not, or restricted to phloem. Finally, the corresponding presence or absence of vertical transmission in plants is considered.

Next, the virus transmission ecologies of the world main livestock viruses, 36 in total, are mapped out, again based on literature data [1]. The extent of virus infiltration in the host-body is held against the duration of infection. Again, viruses are presented in an outer- to inner-body fashion. Considered are four distinct virus organ system tropisms. Viruses colonize epithelia, epithelia plus peripheral nerves and ganglia, epithelia plus reproductive organs system, or also the immune plus circulatory systems. The duration of infection is given as acute, acute and persistent, or persistent. The transmission modes are accordingly fitted, varying from horizontal vertebrate-to-vertebrate transmission to also vertical transmission in vertebrates to also virus transmission via hematophagous arthropods.

Next, the insect specificity of insect transmitted plant viruses is examined in further detail. For this, viruses are grouped by hemipteroid family and corresponding transmission mechanism [7]. The latter may vary from non-persistent, stylet-borne to semi-persistent, foregut-borne to persistent and circulative, retained in the salivary glands, to persistent and propagative, replicating in the insect hemocoel. Insect specificity is considered to be reflected in the virus deep-rootedness and persistence in the insect-body, shift to a single transmission mechanism per insect family, virus replication in the hemocoel, and the shift to vertical transmission in insects.

The intertwined virus life histories and transmission ecologies of plant, arthropod, and vertebrate viruses are accordingly pieced together, based on the virus mono- or instead- dual-host tropism, the location of virus retention or replication on or in the host-body, presence of cyclical or mechanical transmission by arthropods, and the prevailing horizontal or vertical host-to-host transmission modes.

The rationale runs as follows: Published data indicate that arthropod specific viruses evolved into viruses featuring a vertebrate-arthropod-vertebrate cycle and from there evolved into vertebrateonly viruses [5]. The current analysis suggests that some of the latter, rather vertebrate specific, viruses evolved into vertebrate-arthropod-vertebrate, mechanically transmitting viruses, with biting flies or bloodsucking insects acting as vector. Likewise, published data $[5,7]$ indicate that insect specific viruses evolved into viruses featuring a plant-insect-plant transmission cycle. The current analysis identifies these viruses to be highly insect specific. More plant specific viruses evolved into plant-insect-plant, mechanically transmitting viruses. The above four plant-insect-plant transmission mechanisms thus track with a gradient running from highly plant to highly insect specific viruses. 


\section{Results}

Shown in Figure 1 are insect transmitted plant viruses categorized on the basis of the virus retention or replication site on and in the insect-body. The viruses are arranged in an outer- to inner-body fashion and shown along with the virus retention time in the insect and the corresponding virus transmission mechanisms and modes. Also indicated is the virus replication site in the plant host and matching presence or absence of vertical transmission in plants.

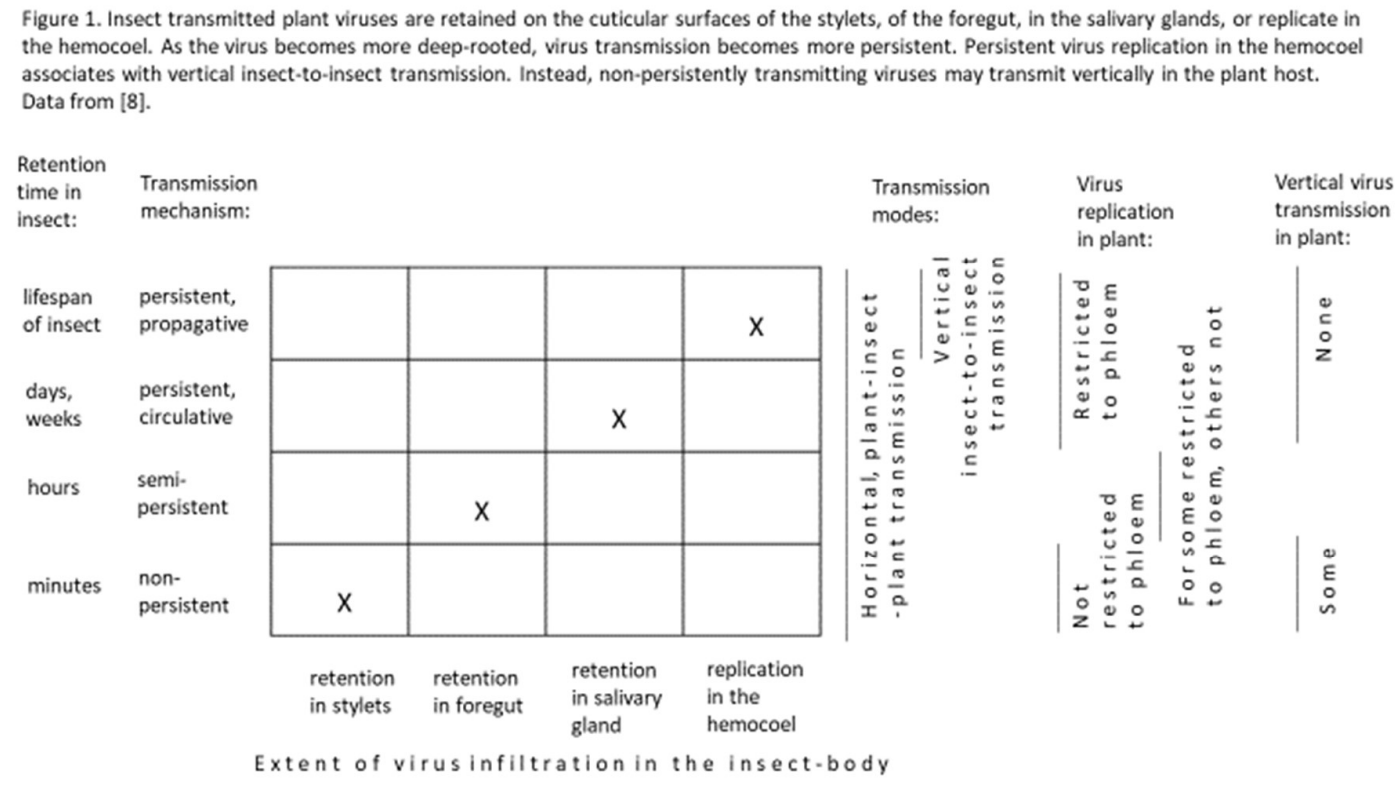

The extent of virus insect-body infiltration and virus transmission persistence are correlated. The utmost deep-rooted, persistent viruses replicate in the insect hemocoel and as a result may be vertically transmitted from insect-to-insect. Instead, some of the non-persistent viruses are vertically transmitted in plants.

Presented in Figure 2, for the world main livestock viruses, 36 in total, is the relationship between the extent of virus infiltration in the host-body and the duration of the infection. Again, viruses are arranged in an outer- to inner-body fashion. The extent of virus host-body infiltration and the duration of infection are correlated. Virus establishment in the reproductive organs system associates with vertical transmission. Viruses circulating in the bloodstream may be transmitted by hematophagous arthropods. 
Figure 2. The world main livestock viruses, 36 in total, are found to colonize epithelia, may establish also latently in peripheral nerves and ganglia, infiltrate the reproductive organs system, or the immune and circulatory systems. As the virus becomes more deep-rooted, the duration of the infection increases. Virus establishment in the reproductive organs system associates with vertical transmission. Viruses colonizing also the immune and circulatory systems and circulating in the bloodstream may become transmitted via hematophagous arthropods. Data from [1].

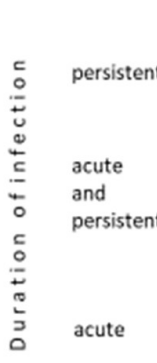

\begin{tabular}{|c|c|c|c|}
\hline & $x$ & $x x x$ & $\begin{array}{l}Y Y Y \\
X X X\end{array}$ \\
\hline $\begin{array}{l}x x x x x \\
x x\end{array}$ & $\begin{array}{l}x x x x x \\
x\end{array}$ & $\begin{array}{l}X X X X X \\
X X X X\end{array}$ & \\
\hline \multicolumn{4}{|l|}{$x x x x$} \\
\hline 1 & 2 & 3 & 4 \\
\hline epithelia & $\begin{array}{l}\text { epithelia plus } \\
\text { peripheral } \\
\text { nerves \& ganglia }\end{array}$ & $\begin{array}{l}\text { epithelia plus } \\
\text { reproductive } \\
\text { organs system }\end{array}$ & $\begin{array}{l}\text { + colonization of } \\
\text { immune and } \\
\text { circulatory systems }\end{array}$ \\
\hline
\end{tabular}

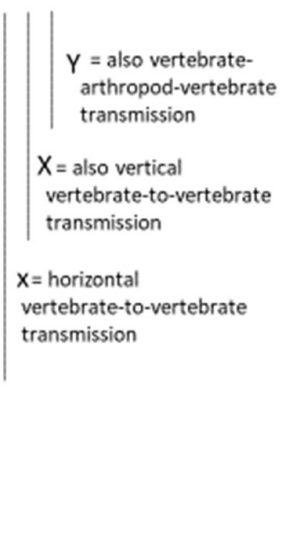

Extent of virus infiltration in the host-body

Figure 3 is best considered in conjunction with Figure 1. Insect transmitted plant viruses are grouped by hemipteroid family and corresponding transmission mechanisms, arranged so that virus deeprootedness and transmission persistence increase from left-to-right. Indicated are the more typical virus genera, with the virus numbers. The relationship thrips-tospovirus, planthopper-tenuivirus, and also planthopper-fijivirus is highly intimate and the virus utmost insect-specific, as implied by the pronounced virus deep-rootedness and persistence, the shift to a single plant-insect-plant transmission mechanism per insect family, by the virus replication in the insect hemocoel, and the associated shift to vertical transmission in insects.

Figure 3. See also Figure 1. Insect transmitted plant viruses grouped by hemipteroid family and corresponding transmission mechanisms, as per Figure 1. Also presented are the major, typical virus genera, with the virus numbers. Towards the top-right corner, the virus becomes increasingly more insect-specific, as implied by the increasing virus deep-rootedness and persistence, shift to a single plant-insect-plant virus transmission mechanism per insect family, virus replication in the hemocoel, and by the shift to vertical transmission in insects. Data from [7].

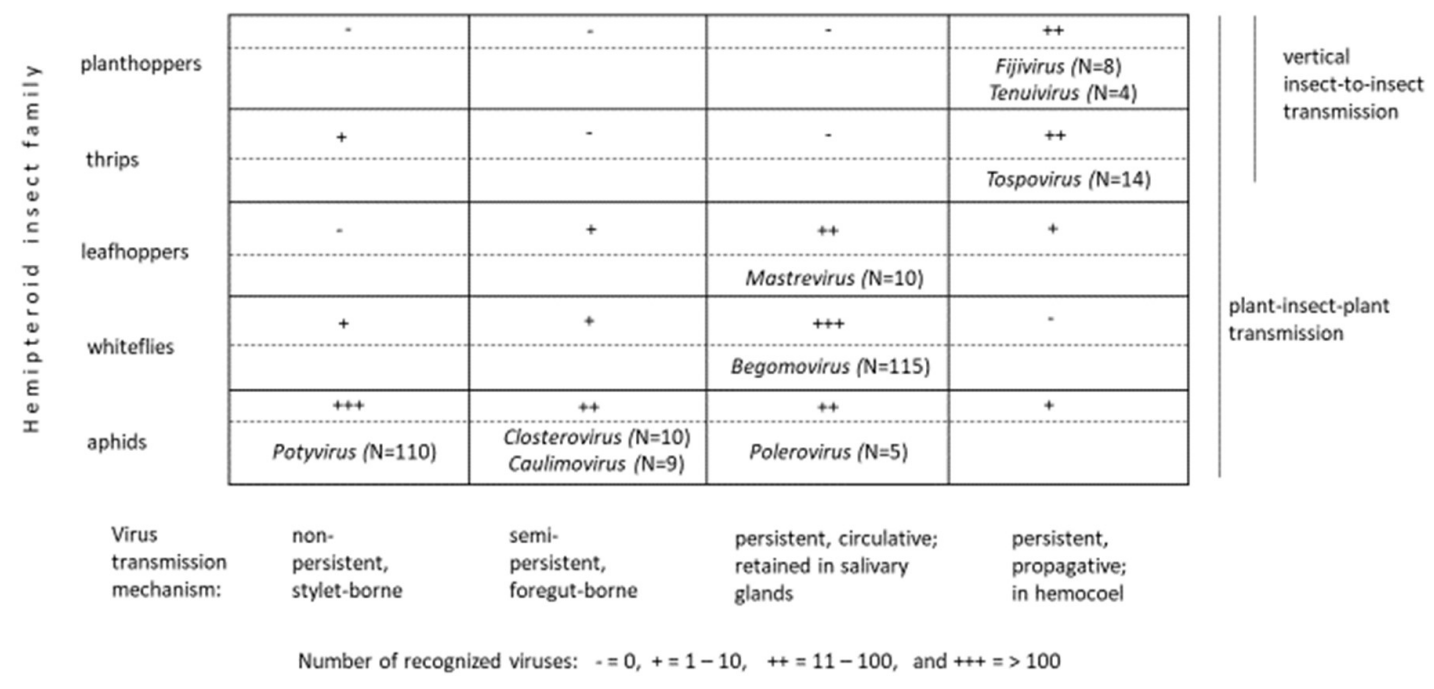

Indicated in Figure 4 are the intertwined plant, arthropod, and vertebrate virus transmission ecologies, best considered in conjunction with Figure 5. Figure 5 provides practical examples of today 
existing virus groupings matching the Figure 4 lay-out. Also indicated in Figure 5 are the inferred virus evolution pathways and links among the virus groupings.

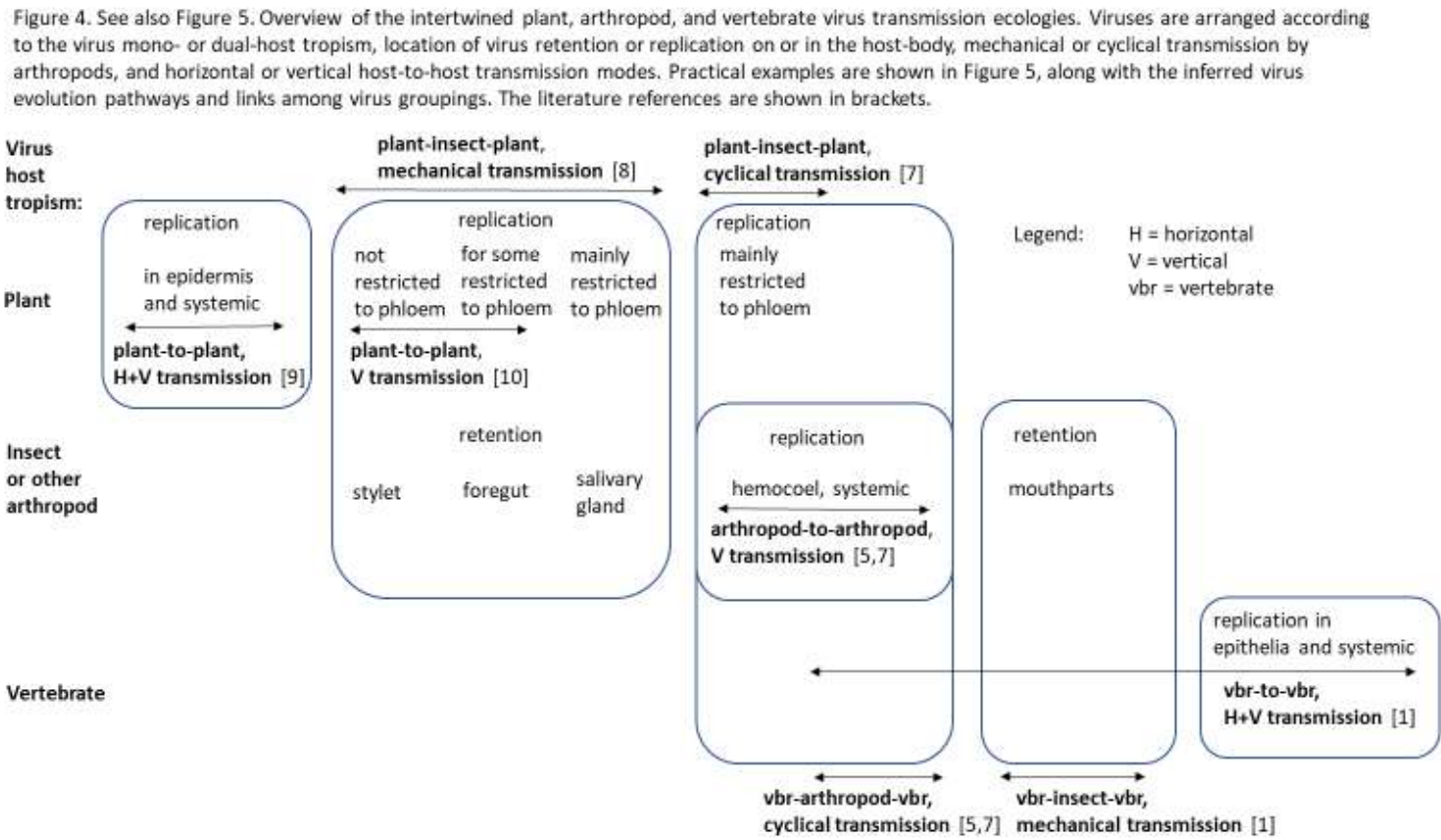

Figure 5. Practical examples of today existing virus groupings matching the Figure 4 lay-out, shown along with the inferred virus evolution pathways and links among virus groupings.

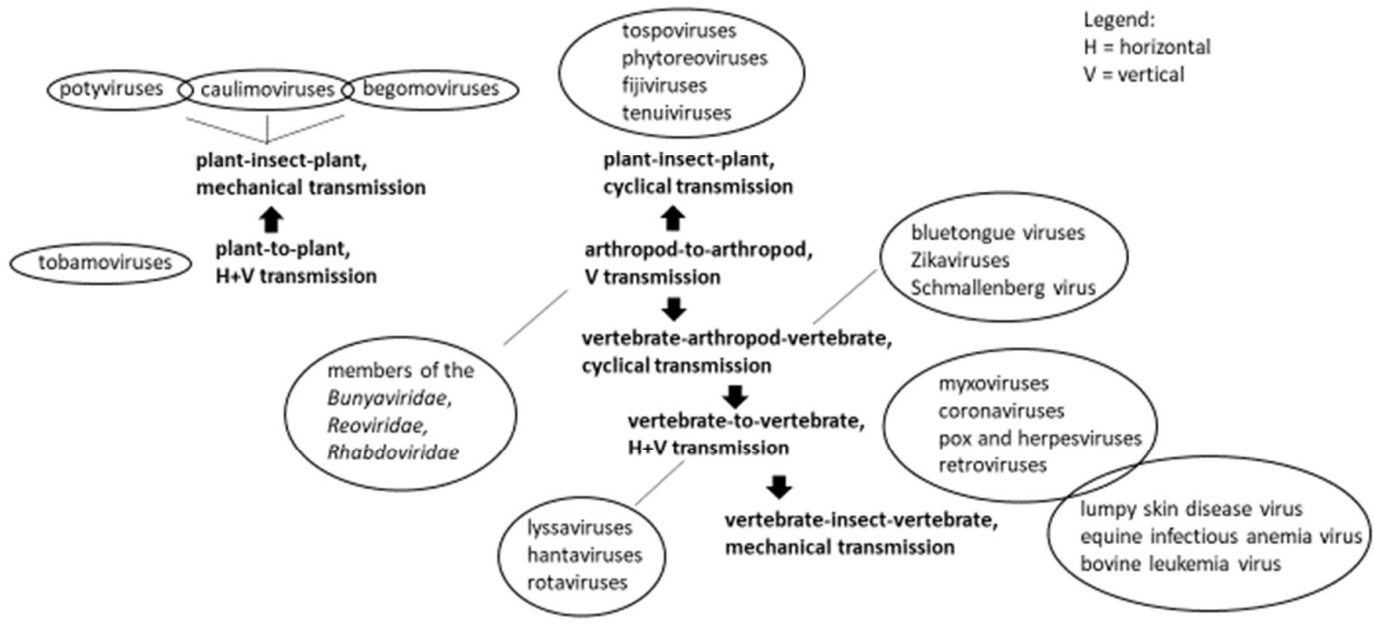

Hematophagous arthropods may transmit an arthropod-specific virus during the bloodmeal and so infect also the vertebrate animal host. Given time, this may translate in a vertebrate-arthropodvertebrate cycle and from there also vertebrate-only viruses may evolve. Some of the vertebrateonly viruses may evolve mechanical transmission modes involving biting flies or bloodsucking insects. The lumpy skin disease virus, the equine infectious anemia virus, and the bovine leukemia virus are examples of viruses transmitting vertically in the vertebrate host, corroborating the vertebrate host origin of these viruses. 
Phytophagous insects may transmit viruses when feeding on phloem sap. Virus replication in the plant phloem does not combine with vertical plant-to-plant transmission. Instead, phloem-sap feeding viruses transmit vertically in insects. Seed transmitting, plant-specific tobamo-like viruses appear to fall basal to plant-insect-plant mechanically transmitted viruses. Some of the potyviruses transmit via seeds. Hence, from tobamovirus to potyvirus to caulimovirus to begomovirus to tospovirus the virus plant-specificity gives way to insect-specificity.

\section{Discussion}

Arthropods and vertebrate animals have more in common with each other than with plants. In both arthropods and vertebrates, virus circulation in the hemocoel or bloodstream associates with vertical transmission. In plants, phloem restricted viruses are highly insect specific and do not transmit via seed. Instead, plant only viruses transmit via seed. This explains why all of today's vertebrate viruses are of arthropod origin and that insect transmitted plant viruses or either of arthropod origin or stem from plant-only viruses. Common to all three host categories is that the vertical transmission mode brings with it a shift towards virus-host mutualism $[2,4,7,11,12]$.

The highly insect-specific bunyaviruses, reoviruses and rhabdoviruses evidently co-evolved with vertebrate animals and plants as host [5,7]. For example, the Maize mosaic virus [MMV], a rhabdovirus, replicates in the nervous tissues of Peregrinus maides, the corn planthopper. In the planthopper the virus infection is more extensive and occurs earlier in the nervous system than in most other tissues. The neurotropic virus route to the salivary glands arguably contributes to the MMV transmission success [7] (pp. 340-342). Strikingly similar rabies viruses are neurotropic and replicate in the salivary glands of carnivore hosts. It thus appears that arthropod rhabdoviruses evolved both into vertebrate-only lyssa viruses, via an intermediary vertebrate-arthropod-vertebrate cycle, and, separately, into the dual-host MMV.

The difference in virus life history and transmission ecology between plant and animal viruses has various plant, animal, and public health protection implications. In the world of plant production, swiftly transmitting tobamoviruses [9] and also potyviruses [10] are of major economic significance because of the damage brought to monoculture crops, and because these viruses seriously complicate global seed trade. Tobamoviruses multiply to very high concentrations and are present in every epidermis cell [14]. Contact-related transmission modes may translate in rapid virus spread [10]. Vertical transmission secures that viruses may survive periods when the host is lacking, become introduced into new areas, are selected and disseminated as host-specific strains, and distributed through the plant population as foci of infection [10].

In the world of livestock production, swiftly transmitting influenza or other myxo-viruses present a challenge to modern poultry and pig mass-rearing. Typically, these opportunistic viruses flexibly adapt the horizontal transmission rate to host abundance. Vertical transmission is more typical for deep-rooted retro- or arboviruses circulating in ruminants, equines, or primates, including humans, as host $[1,4]$.

\section{Funding}

This research received no external funding.

\section{Conflict of interest}

The author declares to conflict of interest. 


\section{References}

1. Slingenbergh, J. Animal Virus Ecology and Evolution Are Shaped by the Virus Host-Body Infiltration and Colonization Pattern. Pathogens 2019, 8, 72. https://doi.org/10.3390/pathogens8020072

2. Villarreal, L.P., Witzany, G. When Competing Viruses Unify: Evolution, Conservation, and Plasticity of Genetic Identities. J Mol Evol 80, 305-318 (2015). https://doi.org/10.1007/s00239-015-9683-y

3. Van Blerkom, L.M. Role of viruses in human evolution. Am. J. Phys. Anthropol 2003, 122, 1446. https://doi.org/10.1002/ajpa.10384

4. Slingenbergh, J. Outer to Inner-body Shifts in the Virus-Host Relationship for the Three Main Animal Host Domains of the World Today: Wildlife, Humans, and Livestock. Immunome Res 2021, 17:186. https://www.longdom.org/open-access/outer-to-innerbody-shifts-in-thevirushost-relationship-for-the-three-main-animal-host-domains-of-the-world-today-wildlifehumans--60230.html

5. Li, C-X; Shi, M.; Tian, J-H; Lin, X-D; Kang, Y-J; Chen, L-J; Qin, X-C; Xu, J.; Holmes, E-C; Zhang, YZ. Unprecedented genomic diversity of RNA viruses in arthropods reveals the ancestry of negative-sense RNA viruses. eLife 2015, 4:05378. https://elifesciences.org/articles/05378.pdf

6. Nault, L.R. Arthropod Transmission of Plant Viruses: A New Synthesis. Annals of the Entomological Society of America 1997, 90, pp. 521-

541. https://doi.org/10.1093/aesa/90.5.521

7. Hogenhout, S.A.; Ammar, E-D.; Whitfield, A.W.; Redinbaugh, M.G. Insect Vector Interactions with Persistently Transmitted Viruses. Annual Review of Phytopathology 2008, 46, pp. 327359. https://doi.org/10.1146/annurev.phyto.022508.092135

8. Mauck, K.E.; Chesnais, Q.; Shapiro, L.R. Chapter Seven - Evolutionary Determinants of Host and Vector Manipulation by Plant Viruses. In Advances in Virus Research, Malmstrom, M., Ed; Academic Press, 2018, Volume 101, pp. 189-250.

https://doi.org/10.1016/bs.aivir.2018.02.007

9. Dombrovsky, A.; Smith, E. Seed Transmission of Tobamoviruses: Aspects of Global Disease Distribution. In Advances in Seed Biology, Jimenez-Lopez, J.C., Ed; Intechopen, 2017. https://www.intechopen.com/books/advances-in-seed-biology/seed-transmission-oftobamoviruses-aspects-of-global-disease-distribution

10. Simmons, H.E.; Munkvold, G.P. Seed Transmission in the Potyviridae. In: Global Perspectives on the Health of Seeds and Plant Propagation Material. Plant Pathology in the 21st Century (Contributions to the 9th International Congress), Gullino M., Munkvold G., Eds.; Springer, Dordrecht, Netherlands, 2014; Volume 6. https://doi.org/10.1007/978-94-017-9389-6 1

11. Roossinck, M.J. Plant Virus Ecology. PLoS Pathog 2013, 9 (5): e1003304. https://doi.org/10.1371/journal.ppat.1003304

12. Simmons, H.E.; Dunham, J.P.; Zinn, K.E.; Munkvold, G.P.; Holmes, E.C.; Stephenson, A.G. Zucchini yellow mosaic virus (ZYMV, Potyvirus): Vertical transmission, seed infection and cryptic infections. Virus Research 2013, 176, pp. 259-264.

https://doi.org/10.1016/i.virusres.2013.06.016

13. Johnson, N.; Phillpotts, R.; Fooks, A.R. Airborne transmission of lyssaviruses. Journal of medical microbiology 2006, 55(6) https://doi.org/10.1099/jmm.0.46370-0

14. Verbeek, M.; Stijger, I.; van der Vlugt, R. Plant Virus Control: The impact of the viruses' transmission strategies. In Prophyta -The Annual 2017, Ruys, T., van Ruiten, J., Timmerman, J., Eds; Global Printing: Velsen, Netherlands, 2017.http://www.prophyta.org/prophyta/Prophyta\%20Annual\%202017.pdf\#page=22 